like fluid. The common bile-duct was traced with great difficulty; for a short distance it was dilated, but it was impervious to the passage of a blowpipe beyond, nor could air be made to pass through the blowpipe into the duodenum. Nevern-square, South Kensington, S.W.

\section{THE EFFECT OF SECTION OF THE PHRENIC NERVES.}

By H. A. haRe, M.D. Univ. of Pennsylvania, DEMONSTRATOR OF THERAPEUTICS AND INSTRUCTOR IN PHYSICAI PENNSYLVANA, PHYSICIAN TO ST. AGNES' HOSPITAL. AND

EDWARD MARTIN, M.D.,

INSTRUCTOR IN OPERATIVF AND CLINICAL SURGERY, UNIVERSITY OF PENNSYLVANIA; SURGEON TO THE PHILADELPHIA AND HOWARD HOSPITALS ; ASSISTANT SURGEON TO THE UNIVERSITY HOSPITAL.

EvERYONE who listens to a course of lectures in a medical institution is told that injuries to the cervical spine, to certain parts of the neck, or to the diaphragm itself, are followed by results of the most serious import, or even by death from failure of respiration owing to paralysis of the diaphragm. Every text-book which is large enough to consider the subject in its breadth teaches the same doctrine ; and while injuries of the kind we have spoken of are not common occurrences, it is essential that, when they do occur, they shall be treated, not as cases in which the prognosis is necessarily fatal, but as instances in which our increasing knowledge may be said to have triumphed over our past ignorance. Everyone knows that respiration, while it is carried on in an identical manner in all warm-blooded animals so far as its major features are concerned, is of a different character in its minor points in nearly every variety of animal life, and, furthermore, in many instances is markedly different even in the two sexes. Thus the respiration of the human being is carried on, not only by the diaphragm, but also by the intercostal muscles and those large bundles of muscle which are so plentifully distributed over the chest wall. It is also well recognised that the movement of the diaphragm and the thoracic respiratory muscles are in unison, and that each one aids the other by increased action whenever necessity arises. That respiration may, however, be carried on sufficiently well to support life, even though these two sets of muscular fibres do not act together, has been proved by such well-known investigators as Mosso, ${ }^{1}$ who found that the want of agreement between the movements of respiration may become so great that the diaphragm and thorax move in opposite directions at the same moment. This, for example, occurs sometimes during the sleep produced by large doses of chloral both in man and the lower animals. Again, it is perfectly possible in some animals to inhibit the action of the diaphragm or thorax completely without producing very marked, or, indeed, any noticeable, change in the general system, either because the action of one is so imperfect, at any rate, that its loss is scarcely felt, or because one portion is enabled by increased action to make compensation for the deficient activity of the other part. A very familiar example of this is seen in every-day life, when some abnormal growth in the abdomen presses upon the diaphragm in such a way as to prevent it from carrying on its functions, and under these circumstances the chest muscles become accustomed to bear a greater strain and to supplement the diaphragm.

Contrariwise, growths in the chest, or chronic disease of the lungs, frequently lead to the total abolition of chest movement and enormous increase in the diaphragmatic functional activity. In the human race we have, perhaps, in a better way than occurs in any of the domestic animals, an example of the variation which sex brings about in the respiratory function. Thus the diaphragm plays a far more important part in respiration in the male than in the female; so that while in man the chest movement is at a minimum and the abdominal movement at a maximum, in the average woman this state of things is directly reversed, for obvious and physiological reasons. In the woman far advanced in pregnancy the upward displacement of the intestines against the diaphragm impedes its movements very greatly, and if the natural breathing of the female was not so largely thoracic, even greater dyspnoea would come on in pregnancy, particularly in its advanced stages, than we are accustomed to see. This form of thoracic respiration is the explanation of the survival of tight corsets in the average woman, and it is the abdominal breathing of the male which makes any similar constriction absolutely unbearable and sufforating. Again, in the female, ascites and all dropsical affections of the abdominal cavity are less frequently associated with dyspnoea than are similar affections in man. Even the enormous ovarian tumours sometimes seen are not accompanied by the dyspnoea one would expect, for the double reason that the thorax moves more easily, and becomes more rapidly accustomed to compensatory action, than does the thorax of the male. Mosso, ${ }^{2}$ however, has made an interesting observation which we have not been able to confirm--namely, that during sleep in both sexes respiration is carried on almost entirely by the thorax, and that in some cases the diaphragm moves so seldom that one might imagine the muscle paralysed. Not only have we failed to note this condition in adult males and females, but also in children, and we think that this statement of Mosso is also at variance with the well. known fact that persons who can lie with the head moderately low during their waking hours cannot lie in the same position without oppression of breathing while asleep. This is doubtless partly due to the fact that voluntaly breathing during sleep is lost; but if Mosso's idea be correct, then the period of sleep would be more comfortable than that of waking, owing to the quietude of the diaphragm, which he believes is normally present when consciousness is removed.

From what we have just said it is evident that woman can physiologically survive injuries affecting the movements of the diaphragm much more readily than can man, and we think that this should be considered as an importan factor when making a prognosis as to the ultimate result of any such traumatism. Those who are cognisant of the recent teaching of physiologists concerning phrenic nerves will remember that their chief function is to innervate the diaphragm, and that, if these nerves are cut or their function is in any way arrested, the diaphragm becomes but a relaxed and flabby sheet of muscle, which is pulled hither and thither as the atmospheric pressure is altered by the movements of the thorax. They will also remember that, according to the most generally received teachings of the past, such a condition has been generally conceded as fatal in man and in most of the lower animals; but we have conclusively proved that this idea is not only fallacious in theory and practice in man, but that it is also partially untrue even in those animals in which respiration depends entirely, during ordinary life, upon the diaphragmatic movements. Thus, in the rabbit of full growth, it will be seen that the chest walls move not at all unless under increased respiratory action; yet we have found, and our results are confirmed by Marckwald, ${ }^{3}$ that section of the phrenic nerves in these animals, provided they are over four months of age, is not necessarily followed by death, and is never so followed provided the animal is allowed to assume a position in which the full play of the chest muscles may be brought about. (See Experiments 1, $2,3,4$, and 5 .)

It is evident, therefore, that in the human being, whose respiratory movements are largely thoracic, there is far less increase in the action of the chest muscles, in order that respiration may be carried on after paralysis of the phrenies, than in an animal whose normal breathing is never thoracic, but always diaphragmatic. Consequently logical reasoning of necessity brings us to the conclusion that injuries producing this lesion should not be fatal in man provided certain precautions are followed.

Although, as has already been stated, all our teachings heretofore have been that such conditions end fatally, we are not without records given us by capable observers proving that our opinion in this matter is correct. Thus Duchenne has observed cases in which complete paralysis and atrophy of the diaphragm existed to such a degree that during inspiration the epigastrium and hypochondrium fell in, while the thorax expanded, the opposite movement occurring during expiration. The patients "appear to

2 Loc. cit.

3 Studies on Respiration, Kreuznach, 1888. 
aspirate their abdominal viscera by dilating their thoraces during inspiration." This same observer states that he has seen this condition in those suffering from hysteria, progressive muscnlar atrophy, and chronic lead poisoning, and that he is cognisant of cases in which this condition persisted for years. Op.r reasoning that man should, theoretically, suffer less than rabbits from diaphragmatic palsy is supported, therefore, both by clinical evidence and also by experiments which we have recently performed, the results of which form part of this paper.

Returning for one moment in order that we may adduce still further clinical evidence in support of our views, we may mention a case described by Bell, in which a person with injury to the spinal cord above the origin of the phrenic nerves survived the accident for half an hour. That life was not prolonged for a greater period was due, in this case, to the fact that not only the phrenic nerves, but, in addition, all the nerves supplying the muscles of the chest, were paralysed by the injury, so that thoracic movement was absolutely impossible. This is evidenced by the statement of Bell himself, who asserts that the movements of respiration were carried on solely by contractions of the muscles of the neck and shoulders. We have found that in some of the higher animals whose breathing is largely thoracic, no untoward effects follow phrenic section, and Panizza has done lfkewise; thus, in the dog, while paralysis of the diaphragm produces instantly very noticeable changes in the respiratory movements, it cannot be said that any signs of asphyxia develop themselves, simply because the thoracic muscles instantly increase their functional activity. That this increase in activity is not only imaginary, but real, is proved by the fact that if the animal be killed several weeks later, the muscles of the chest are found greatly hypertrophied, and the antero-posterior and lateral diameters of the thorax are greatly increased. At the same time it is to be noted that the diaphragm has undergone very marked degeneration and atrophy as a result of its lack of nerve-supply and functional activity. A very typical instance showing this result can be obtained in the animal in which the phrenic nerve has been cut on one side only, as under these circumstances the half of the diaphragm on the injured side may, in the course of two weeks, be found thin and membranous, while the opposite side not only preserves its normal thickness, but is generally better developed than before. We have performed bilateral phrenic section in the rabbit, the dog, the goat, the horse, and the monkey, and the results reached we now wish to place before our readers. (See Experiments 1, 2, 3, 4, and 5 ) In order that no more pain might be given than was necessary, and chiefly to avoid a reopening of the wound for antiseptic reasons, we have found it necessary to make section of these nerves, not by the scissors or knife, but by a sudden quick jerk of the hitherto slack ligature passed behind the nerve. If the jerk be instantaneous and forcible, a fine, strong ligature cuts its way through with less pain to the animal than would ordinarily be imagined; and while, at the first glance, it would seem as if one could not tell that the nerves were completely severed by such means unless the wound was opened, yet the mere fact that a loop comes away perfectly intact, and often with a small quantity of nervous matter adhering to it, proves absolutely that the nerve has been completely divided.

(To be concluded.)

\section{THE TREATMENT OF FRACTURE OF THE PATELLA BY MAYO ROBSON'S METHOD.}

BY J. FLETCHER HORNE, F.R.C.S. ED., SURGEON TO THE BARNSLEY BECKETT HOSPITAL.

THE treatment of simple transverse fracture of the patella from the sixteenth century to the present time has been a pons asinorum to the surgeon, as evidenced by the variety of treatment-trom the incline plane, pulley and weight, Malgaigne's hooks, to laying open the joint and wiring the fragments together. Thomas of Liverpool has said: "Of all the important fractures presented to the surgeon for treatment, the easiest to restore and repair is fracture of the patella." This I venture to think is not the verdict of the profession generally; it may be easy to treat, but of all fractures the most difficult to get bony union. Hamilton, without denying the possibility of union by bone, says: "I do not know positively that in any case the union was by bone"; whilst Hutchinson writes that no proof has yet been afforded that transverse fracture of the patella with considerable separation at the time ever unites by bone. I find the Musée Dupuytren and the Museum of the Royal College of Surgeons of Ireland have each specimens of true osseous union. In a large majority of cases under any plan of treatment a fibrous union of the fragments is all that can be expected; to obtain the shortest possible ligamentous union, possibly an osseons one, is the object of our ambition; for its attainment I think the plan advocated by my friend, Mr. A. W. Mayo Robson, will be found one of the best of the many which have been devised for approximating the fragments by direct pressure.

Soon after reading a short résumé in THE LANCET of June 1st, 1889, of Mr. Robson's paper, read before the Clinical Society of London, May 24th, 1889, I was called to the case of which I append the following notes.

M. S - - aged thirty, miner, on July 6 th, at 2 P. M., walking home from work, caught his foot upon a bit of stone on the footpath, dropped on his right knee and then upon his face. He felt very sick, was raised by his companions, and, as he was unable to walk, they carried him a short distance, and then got him home in a waggonette. I saw him at 5 o'clock, his legs stretched across two chairs. There was complete loss of power over the joint, with considerable pain and swelling. The fracture was easily made out as a transverse one, the fragments separated to the extent of an inch, with the limb extended. The patient was placed under ether by my colleague, Dr. Wetherell, the limb washed, and then carefully bathed with bichloride of mercury lotion ( 1 in 1000). I procured two steel pins seven inches long, with large glass heads, as used by ladies to fasten on the bonnet, and carefully purified them. I passed one through the tendon of the quadriceps extensor, just above the superior edge of the upper fragment, the second needle being passed in like manner through the ligamentum patella. By traction the fragments of bone were easily brought into apposition. A figure-of-eight ligature of fine whipcord was passed over the pins to keep the fragment of bone in position, and the sharp points of the pins cut off. The cut ends were guarded by cotton-wool and the joint encased in a plaster-of-Paris bandage. As the aspirator was not at hand, I omitted its use to draw off the effusion as recommended. The temperature never rose above normal, and on July 27th, three weeks having elapsed, a window; was cut in the bandage large enough to withdraw the pins. A slight oozing of blood followed, which was mopped up with carbolised wool, dusted with iodoform, and the plaster-of-Paris reapplied. On Aug. 27th the limb was cleared of the bandage, the fracture found in good position, and placed on Arnold's knee splint with exten sion screw, held in position by plaster-of-Paris bandage, and the patient allowed to get about on crutches; a privilege of which he availed himself readily. On Nov. 23rd the splint was removed; union appeared to be bony and complete, the case having been under treatment twenty weeks. On Dec. 12th I examined the knee. No change had taken place from freely walking out each day. The patient certainly walked well, showing no stiffness or deformity. The treatment differed slightly from Robson's in minor details, as I had to trust to memory, but the broad principle remains. Its advantages appear to be simplicity, the absence of risk, and the obtaining of bony union.

Gux's HospitaL. - A gratifying and tangible recognition of the services of many of the nurses took place at this hospital last week, by the presentation to each of them of a medal--a graceful decoration of merit which might be adopted by other similar institutions.

Naval College, Portsmouth.-In consequence of the late prevalence of typhoid fever at this College, and a suspicion of the existence of sanitary defects as the cause, the drainage of the institution has been submitted to scrutiny by experts, who, however, have failed to detect anything so faulty as to warrant the conclusion that the epidemic had arisen from such a source. 